\title{
Broad-RBFNN-Based Intelligence Adaptive Antidisturbance Formation Control for a Class of Cluster Aerospace Unmanned Systems with Multiple High-Dynamic Uncertainties
}

\author{
Erxin Gao $\mathbb{D}^{1},{ }^{1,2,3}$ Xin Ning $\mathbb{D}^{1,3}$ Zheng Wang $\mathbb{D}^{3,4}$ and Xiaokui Yue $\mathbb{D}^{1,3}$ \\ ${ }^{1}$ School of Astronautics, Northwestern Polytechnical University, Xi'an 710072, China \\ ${ }^{2} X i$ 'an Institute of Electromechanical Information Technology, Xi'an 710 065, China \\ ${ }^{3}$ National Key Laboratory of Aerospace Flight Dynamics, Northwestern Polytechnical University, Xi'an, China \\ ${ }^{4}$ Research Center for Unmanned System Strategy Development, Northwestern Polytechnical University, Xi'an, China
}

Correspondence should be addressed to Xin Ning; nx_nwpu@163.com

Received 26 December 2020; Revised 6 November 2021; Accepted 18 November 2021; Published 7 December 2021

Academic Editor: Xianggui Guo

Copyright (c) 2021 Erxin Gao et al. This is an open access article distributed under the Creative Commons Attribution License, which permits unrestricted use, distribution, and reproduction in any medium, provided the original work is properly cited.

\begin{abstract}
This paper investigates the antidisturbance formation control problem for a class of cluster aerospace unmanned systems (CAUSs) suffering from multisource high-dynamic uncertainties. Firstly, to estimate and compensate the uncertainties existing in CAUS coordinate dynamics, an adaptive antidisturbance formation control law, which is combined by a robust adaptive control law and the second order disturbance observer, has been designed. Secondly, aiming at the adverse influences caused by the nonlinear time-varying nonlinearities existing in the formation flight dynamics, the radial basis function neural network (RBFNN) is introduced. Furthermore, considering the rapidly varying characteristics of the aforementioned formation flight nonlinearities, a novel board RBFNN (B-RBFNN) has been constructed and utilized to improve the approximation and compensation performance. In virtue of the fusing of the B-RBFNN and the second-order disturbance observer-based adaptive formation control law, the rapid response rate and the higher control accuracy of the formation control system can be achieved. As a result, a novel B-RBFNN-based intelligence adaptive antidisturbance formation control algorithm has been established for CAUS trajectory coordination and formation flight. Numerical simulation results are proposed to illustrate the effectiveness and advantages of the proposed B-RBFNN-based intelligent adaptive formation control method for the CAUS.
\end{abstract}

\section{Introduction}

The aerospace unmanned systems (AUSs) are a class of power-driven, unmanned, reusable aircrafts that possess a series of advantages, including low cost, long duration of combat, and zero casualties. Currently, AUSs are widely used in a variety of combat missions, such as ground attack, communication relay, and target searching and tracking. When performing combat missions in high-risk environments, AUSs may demonstrate greater advantages [1]. In order to improve the ability of AUSs for completing complex and high-difficulty tasks, many researchers have carried out the study of cluster aerospace unmanned systems (CAUS). In the past decade, the CAUS technology has become one of the research hotspots, and the researchers have proposed a series of effective methods for task assignment, trajectory planning and formation control of the CAUS [2-4].

Formation control is of great importance in the research of CAUS. A perfect formation can effectively avoid obstacles without complex formation transformation and can modify the overall endurance time of the CAUS. In practical, formation shape and maintenance of the CAUSs cannot be separated from the support of formation control algorithm. At present, a plenty of formation control methodologies, including graph theorybased method [5], leader-follower method [6], behavioral-based method [7], artificial potential field method [8], and virtual structure method [9], have been extensively investigated for the CAUS. Graph theory can describe the topology of 
communication between multiple agents and can be used to model the whole multiagent. By utilizing this feature, Marcio et al. presented a new formation control law based on rigid graph theory, which can achieve stable formation control for largescale CAUS [10]. Jongho et al. took full advantage of the fact that the behavior-based formation control method does not require the AUS in the cluster to have global information, designed a behavior-based formation controller based on the position and speed of the adjacent AUS, ensuring the uniform stability of the closed-loop system [11]. Inspired by the behavior of pigeons, Qiu et al. proposed a formation control method based on behavior characteristics of pigeons, using the potential field function theory to define the topological structure and the master-slave relationship in the group, thus realizing the formation flight of the CAUS [7]. Askari et al. used the virtual structure method to introduce the formation feedback algorithm into formation control of CAUS, which improved the accuracy of maneuver [12]. Most recently, the leader-follower method is recognized as one of the most commonly used methods in the field of formation control of CAUS. Turpin et al. improved the leaderfollower formation method and adopted a distributed communication mode instead of using the leader-follower direct communication with all the followers [13]. By utilizing airborne visual perception equipment to obtain the position information of the CAUS, Saska et al. adopted the leader-follower method to realize the formation flight of the CAUS under non-GPS positioning [14]. Considering the shortcoming that the traditional leader-follower method cannot unify formation control and obstacle avoidance, Shao et al. presented a formation control method of CAUS by combining leader-follower method with artificial potential field method and achieved stable formation control with obstacle avoidance ability [15].

In practical, the suppression or compensation for the multiple disturbances is essential to improve the control performance of the practical engineering system. Thus, the researches on disturbance suppression or compensation have spurred considerable attentions in recent years. The traditional antidisturbance control mainly relies on the robustness of the control law. These methods mainly include sliding mode control $[16,17]$, adaptive control $[18,19]$, and $H_{\infty}$ control $[20,21]$. With the development of disturbance rejection control technology, the disturbance observer-based control (DOBC) $[22,23]$ and the active disturbance rejection control (ADRC) $[24,25]$ have gradually appeared. Recently, the DOBC has been greatly developed. A class of nonlinear disturbance observer is proposed in literature [26] and applied to the design of missile attitude control law. [27-29] discusses the application of antidisturbance control method based on the disturbance observer in industrial and mechanical systems. Considering that multilevel and complex interference often occurs in practical applications, literature [30] proposed a combined layered anti-interference control method, which is extended to Markov jumping system [31], higher-order system [32] and fuzzy system [33]. The authors of [34] study the estimation and compensation methods for a class of nonlinear dynamic disturbances. The authors of [35] discussed the combined antidisturbance control method for a class of fuzzy random nonlinear systems and studied the disturbance estimation and suppression methods for a class of Markov uncertain systems. Lately, some antidisturbance methods are implemented in the design of attitude control of AUS. Zhu et al. [36] used ADRC to solve the problem that small fixed-wing AUS is vulnerable to wind interference when landing. In [37], a combination algorithm of DOBC and $H_{\infty}$ is exploited, which is able to carry out strong robust control over the quadrotor AUS subject to external disturbance. In [38], the combined algorithm of $\mathrm{ADRC}$ and $\mathrm{DOBC}$ can better solve this problem and greatly improve the stability.

In spite of the progress, most of the above formation control works only focused on the cluster systems subject to a single-source and slow-varying disturbance. However, in the actuator flight process of the CAUS, the multiple disturbances, including the unmodeled dynamics, the winds, the aerodynamic uncertainties, and the input and structural uncertainties, are inevitable. When AUS operates in complex scenes, the source and type of disturbances are usually not unique, and the high-dynamic disturbance may be encountered. If the multisource and high-dynamic features are ignored in the formation control law design, the actual flight paths of the CAUS may be different from the nominal ones, and the failed tasks may be caused. Therefore, it can be concluded that although many excellent results have been obtained for CAUS, there has been no study on the trajectory coordination and formation control for the CAUS under the multisource high-dynamic uncertainties. Moreover, the rapid response rate of the formation control system is one of the key indexes for CAUS. In [39], a novel effective learning strategy-board learning system (BLS) - has been proposed, which can be utilized to improve the response rate of the formation control system. However, so far, the board learning thought has never been applied to the formation control of the CAUSs, and the research of BLS-based rapid formation control structure has to be developed.

In this paper, a novel intelligence adaptive antidisturbance formation control has been proposed for CAUSs based on B-RBFNN. A second order disturbance observer and a robust adaptive control law are designed to estimate and compensate the multisource high-dynamic uncertainties. To deal with the nonlinear time-varying nonlinearities existing in the formation flight dynamics, the board RBFNN is utilized as online learning system in the control structure. Compared with the existing literature, the main contribution of this paper can be concluded as follows:

(1) In this paper, a novel B-RBFNN based intelligence adaptive antidisturbance formation control method has been proposed for CAUS with multiple uncertainties

(2) Combining the B-RBFNN and the second-order disturbance observer, the rapid response rate and the higher control accuracy of the formation control system can be guaranteed

(3) This paper provides a board learning system-based intelligence adaptive formation control canonical form for the cluster systems, which can be applied to cluster underwater unmanned systems, cluster space unmanned system and so on 


\section{Problem Formulation and Preliminaries}

2.1. The System Model of CAUS. According to [40], we can get the kinematics and dynamics equations of the $i$-th $(i=$
$1,2, \ldots, N)$ AUS in the flightpath-coordinate-system as follows:

$$
\left\{\begin{array}{l}
\dot{x}_{i}=v_{i} \cos \theta_{i} \cos \psi_{s, i}, \\
\dot{y}_{i}=v_{i} \sin \theta_{i}, \\
\dot{z}_{i}=v_{i} \cos \theta_{i} \sin \psi_{s, i}, \\
m_{i} \dot{v}_{i}=P_{i} \cos \alpha_{i} \cos \beta_{i}-Q_{i}-m_{i} g \sin \theta_{i}+d_{i, 1}, \\
m_{i} v_{i} \dot{\theta}_{i}=P_{i}\left[\cos \alpha_{i} \sin \beta_{i} \sin \gamma_{s, i}+\sin \alpha_{i} \cos \gamma_{s, i}\right]+Y_{i} \cos \gamma_{s, i}-Z_{i} \sin \gamma_{s, i}-m_{i} g \cos \theta_{i}+d_{i, 2}, \\
-m_{i} v_{i} \cos \theta_{i} \dot{\psi}_{s, i}=P_{i}\left[-\cos \alpha_{i} \sin \beta_{i} \cos \gamma_{s, i}+\sin \alpha_{i} \sin \gamma_{s, i}\right]+Y_{i} \sin \gamma_{s, i}+Z_{i} \cos \gamma_{s, i}+d_{i, 3} .
\end{array}\right.
$$

where $x_{i}, y_{i}, z_{i}$ are the positions in three directions for the $s$-th AUS; $v_{i}$ represents the velocity; $\theta_{i}, \psi_{s, i}$ are the flight path angle and the flight path deflection angle; $\alpha_{i}, \beta_{i}, \gamma_{s, i}$ are the angle of attack, angle of sideslip and the heeling angle respectively; $P_{i}$ is the thrust, $Q_{i}$ is the drag, $Y_{i}$ is the lift-force, $Z_{i}$ is the side-force; $d_{i, 1}, d_{i, 2}, d_{i, 3}$ denote the external disturbances, $m_{i}$ represents the mass, and $g$ is the acceleration of gravity.

In practical, the influence of the multiple uncertainties and disturbances are unavoidable. Considering the composite uncertainties, the dynamic equations of the CAUS can be rewritten as

$$
\left\{\begin{array}{l}
\dot{x}_{i, 1}(t)=\left(A_{i}(t)+\Delta A_{i}(t)\right) x_{i, 2}(t) \\
\dot{x}_{i, 2}(t)=g\left(\alpha_{i}, \beta_{i}, \gamma_{s, i}\right) u_{i}(t)+f\left(\theta_{i}, v_{i}\right)+\Delta f\left(\theta_{i}, v_{i}\right)+d_{i}(t), \\
y_{i}(t)=x_{i, 1}(t)
\end{array}\right.
$$

where $x_{i, 1}=\left[x_{i}, y_{i}, z_{i}\right]^{T}, x_{i, 2}=\left[v_{i}, \theta_{i}, \psi_{i, s}\right]^{T},(i=1,2, \ldots, n)$ represent the system state vector; $y_{i}(t) \in \mathbb{R}^{3 \times 1}, u_{i}(t) \in \mathbb{R}^{3 \times 1}$ denote the control input and output vector, respectively; $d_{i}(t) \in \mathbb{R}^{3 \times 1}$ is the external disturbances; $f\left(\theta_{i}, v_{i}\right) \in \mathbb{R}^{3 \times 1}$ is the known bias vector; $\Delta f\left(\theta_{i}, v_{i}\right) \in \mathbb{R}^{3 \times 1}$ represents an unknown smooth function. And, $A_{i}(t) \in \mathbb{R}^{3 \times 3}$ is control system parameter matrices with proper dimensions, and $\Delta A_{i}(t)$ represents the structural uncertainties of $A_{i}(t)$; $g\left(\alpha_{i}, \beta_{i}, \gamma_{s, i}\right) \in \mathbb{R}^{3 \times 3}$ denotes control distribution matrix. The aforementioned matrices can be provided as

$$
\begin{aligned}
& A_{i}(t)=\left[\begin{array}{ccc}
\cos \theta_{i} \cos \psi_{s, i} & -v \sin \theta_{i} \cos \psi_{s, i} & -v_{i} \cos \theta_{i} \sin \psi_{s, i} \\
\sin \theta_{i} & v_{i} \cos \theta_{i} & 0 \\
-\cos \theta_{i} \sin \psi_{s, i} & v_{i} \sin \theta_{i} \sin \psi_{s, i} & -v_{i} \cos \theta_{i} \cos \psi_{s, i}
\end{array}\right] \\
& f\left(\theta_{i}, v_{i}\right)=\left[\begin{array}{c}
\frac{\left(-Q_{i}-g \sin \theta_{i}\right)}{m_{i}} \\
\frac{\left[P_{i}\left(\cos \alpha_{i} \sin \beta_{i} \sin \gamma_{s, i}+\sin \alpha_{i} \cos \gamma_{s, i}\right)-Z_{i} \sin \gamma_{s, i}-m_{i} g \cos \theta_{i}\right]}{m_{i} v_{i}} \\
-\frac{\left[P_{i}\left(-\cos \alpha_{i} \sin \beta_{i} \cos \gamma_{s, i}\right)+Z_{i} \cos \gamma_{s, i}\right]}{m_{i} v_{i} \cos \theta_{i}}
\end{array}\right], \\
& g\left(\alpha, \beta, \gamma_{s}\right)_{i}=\left[\begin{array}{ccc}
\cos \alpha_{i} \cos \beta_{i} & 0 & 0 \\
0 & \left(C_{y}^{\alpha}\right)_{i} \cos \gamma_{s, i} & 0 \\
0 & 0 & P_{i} \sin \alpha_{i} \cos \gamma_{s, i}+Y_{i} \cos \gamma_{s, i}
\end{array}\right] .
\end{aligned}
$$


Remark 1. CAUS will inevitably be affected by high dynamic disturbance in actual flight process. To be specific, the wind, which is the CAUSs suffered, includes constant wind, dynamic wind, discrete abrupt wind, and shear wind, and the wind speed may reach $15 \mathrm{~m} / \mathrm{s}$, which will have a significant impact on the CAUS's flight stability. In addition, aerodynamic disturbances, fabrication process, installation errors, and control system errors (instrument errors, actuator errors) are all sources of matched uncertainties.

In order to make further discussion, it is necessary to give the following assumptions.

Assumption 1. It is assumed that the unknown disturbances rate is bounded. For $i=1,2, \ldots, N$, there exists a positive constant $\mu$, such that $\left\|\dot{d}_{i}\right\| \leq \mu$ and $\left\|\ddot{d}_{i}\right\| \leq \mu$.

Assumption 2. It is assumed that the uncertainties of cannot change the control directions, that is, $\lambda_{\max }\left(\Omega_{i}\right)>0$, where $\Omega_{i}=\left(A_{i}(t)+\Delta A_{i}(t)\right) \cdot A_{i}^{-1}(t)$.

Therefore, the goal of this paper is to design an intelligence adaptive antidisturbance formation control algorithm for the CAUS (2) to achieve the position coordination and system stability under the existence of external disturbances and input uncertainties.

2.2. Algebraic Graph Theory. For the CAUS formation controller design, the algebraic graph theory is necessary. Let $G=(V, E, A)$ represents a weighted digraph and $G$ is used to model the communication network among the agents, where $V=\left\{v_{1}, v_{2}, \ldots, v_{n}\right\}$ represents the set of nodes, $E \subseteq V \times V$ represents the set of the edges, and $A=\left[a_{i j}\right]$ represents the adjacency matrix. Additionally, the node $v_{i}$ denotes the $i$ th agent. The edge of the weighted graph $G,(i, j) \in E$ is represented by the edge $(i, j)$ if and only if there is a communication from agent $j$ to agent $i$. The neighbor set of node $v_{i}$ can be described as $N_{i}=\left\{v_{j} \mid(i, j) \in E\right\}$. And, the communication quality between the agents $i$ and $j$ is represented by the adjacency element $a_{i j}$, which is corresponding to the edge $(i, j)$, i.e., $(i, j) \in E \Leftrightarrow a_{i j}>0$; otherwise, $a_{i j}=0$. A weighted graph $G$ is called undirected if and only if $a_{i j}=a_{j i}$. Undoubtedly, $A$ is symmetric and the diagonal elements $a_{i i}=0$ for undirected weighted graphs. Moreover, the foundation of algebraic graph theory can be referenced in literature [41].

\subsection{Broad Radial Basis Function Neural Network.} According to [41], by the use of NNs, the uncertainty and the disturbance in the nonlinear systems can be estimated. Thus, RBFNN is brought in for approximation. For any given continuous function $f(x): \mathbb{R}^{n} \longrightarrow \mathbb{R}$ on a compact set $U \in \mathbb{R}^{n}$ and an arbitrary $\varepsilon>0$, there exists the RBFNN $\Theta^{T} \Phi(x)$ such that $\sup _{x \in U}\left|f(x)-\Theta^{T} \Phi(x)\right|<\varepsilon$, where $\Theta \in \mathbb{R}^{l}$ is the weight vector, $l$ is the node number, $x \in \mathbb{R}^{n}$ is the input vector, and $S(x)=\left[s_{1}(x), \ldots, s_{l}(x)\right]^{T}$ is Gaussian basis function vector, which can be expressed by

$$
s_{i}(x)=\exp \left(-\frac{\left\|x-\pi_{i}\right\|^{2}}{\omega_{i}^{2}}\right), \quad i=1, \ldots, l,
$$

where $\pi_{i}=\left[\pi_{i, 1}, \ldots, \pi_{i, n}\right]^{T}$ and $\omega_{i}$ represents the center and the width of the Gaussian function, respectively. In the ideal condition, we can get the following equality:

$$
f(x)=\left(\Theta^{*}\right)^{T} \Phi(x)+\varepsilon^{*},
$$

where $\Theta^{*}$ represents the optimal weight vector and $\varepsilon^{*}$ represents the approximation error. In conclusion, $\Theta^{*}$ and $\varepsilon^{*}$ are bounded. In the process of controller design, the unknown functions of the systems and the uncertain parameters are unaccessible.

In [39], as an alternative way for deep learning and structure, broad learning system (BLS) has been proposed to offer an idea of extension in width structure. In a BLS, we first map the input vectors to construct feature nodes by feature mappings. Secondly, the mapped features are concatenated to take nonlinear transformation so that the enhancement nodes are formed. Furthermore, connect the whole outputs of mapped features and enhancement nodes into the output layer.

It can be assumed that we present the input data $X$ and project the data, using $\phi_{i}\left(X W_{e i}+\beta_{e i}\right)$, to become the $i$ th mapped features, $Z_{i}$, where $W_{e i}$ is the random weights with the proper dimensions. Denote $Z^{i} \equiv\left[Z_{1}, \ldots, Z_{i}\right]$ which is the connection of all the first $i$ groups of mapping features. Similarly, the $j$ th group of enhancement nodes, $\xi_{j}\left(Z^{i} W_{h j}+\right.$ $\beta_{h j}$ ) is denoted as $H_{i}$, and the connection of all the first $j$ groups of enhancement nodes are represented as $H^{j}=\left[H_{1}, \ldots, H_{j}\right]$. Hence, the broad model can be represented as follows:

$$
\begin{aligned}
Y & =\left[Z_{1}, \ldots Z_{n} \mid \xi\left(Z^{n} W_{h 1}+\beta_{h 1}\right), \ldots, \xi\left(Z^{n} W_{h m}+\beta_{h m}\right)\right] W^{m} \\
& =\left[Z_{1}, \ldots Z_{n} \mid H_{1}, \ldots, H_{m}\right] W^{m} \\
& =\left[Z_{n} \mid H_{m}\right] W^{m} .
\end{aligned}
$$

where $W^{m}=\left[Z^{n} \mid H^{m}\right]^{+} Y . \quad W^{m}$ denotes the connecting weights for the broad structure.

Undoubtedly, we can use BLS to expand the structure of RBFNN when new feature nodes and enhancement nodes are needed. Meanwhile, the expanded structure will possess more excellent approximation ability. In the next section, we will use the B-RBFNN to estimate these functions, and the weight matrices of the B-RBFNN will be approximated by designing several adaptive laws.

\section{Main Result}

The proposed control scheme can be divided into the inner loop and outer loop. In the inner loop, in order to overcome the input uncertainty in the collaborative flight of AUS, an adaptive law is designed. In the outer loop, B-RBFNN is applied for approximation in view of the time-varying nonlinearities. To deal with the time-varying disturbances $d_{i}$, a second-order disturbance observer is proposed, which can 
both estimate $d_{i}$ and the derivative $\dot{d}_{i}$ synchronously. Meanwhile, $\dot{d}_{i}$ will be fed back into the equation of the disturbance estimation, reducing the estimation error caused by the disturbance change rate. At last, the estimated disturbance values are introduced into the formulation control law, and a coordinate adaptive antidisturbance control structure is constructed. The proposed control structure can be depicted by Figure 1 .

3.1. Input-Uncertainty-Suppressed Inner Loop Adaptive Formation Control Law Design. According to the consistency theory [41], we can define the tracking error as

$$
\begin{aligned}
& Z_{i, 1}=\sum_{j=1}^{N} a_{i j}\left(y_{i}-y_{j}\right)+b_{i}\left(y_{i}-y_{r}\right), \\
& Z_{i, 2}=x_{i, 2}-\beta_{i, 1},
\end{aligned}
$$

where $a_{i j}$ represent the elements in adjacency matrix $A$ and $b_{i}$ represent the elements in information connection matrix $B$ of the AUSs and their leader.

Define the inner loop system error $e_{y i}=y_{i}-y_{r}$, where $y_{r}$ represents the leader state. According to (3), we can take the differential of $e_{y i}$ as

$$
\begin{aligned}
\dot{e}_{y i} & =\dot{y}_{i}-\dot{y}_{r} \\
& =\left(A_{i}(t)+\Delta A_{i}(t)\right) x_{i, 2}(t)-\dot{y}_{r} .
\end{aligned}
$$

Define $\Omega_{i}=\left[A_{i}(t)+\Delta A_{i}(t)\right] / A_{i}(t)$. Thus, we can rewrite (8) as

$$
\dot{e}_{y i}=A_{i}(t) \Omega_{i} x_{i, 2}(t)-\dot{y}_{r} .
$$

Therefore, the indirect virtual control signal is designed as

$$
x_{i, 2 c}(t)=A_{i}^{-1}(t)\left(-K_{1} Z_{i, 1}+\dot{y}_{r}\right),
$$

where $K_{1} \in \mathbb{R}^{3 \times 3}$ represents the control gain of the inner loop.

However, it is obvious that because of the effect of uncertainty term $\Delta A_{i}(t)$, which cannot achieve the stability of the inner loop system only use the control signal $x_{i, 2 c}(t)$, so we propose the final virtual control signal in the inner loop as $\beta_{i, 1}$, which is designed as

$$
\beta_{i, 1}=x_{i, 2 c}(t)+x_{i, 2 a}(t),
$$

where $x_{i, 2 a}(t)$ is the adaptive compensation term of the input uncertainty, which can be obtained that

$$
x_{i, 2 a}(t)=-\widehat{\Psi}_{i} x_{i, 2 c}(t),
$$

where $\widehat{\Psi}_{i}$ is the estimation of $\Psi_{i}$, and $\Psi_{i}=\left(\Omega_{i}-I_{3}\right) \cdot \Omega_{i}^{-1}$.

Moreover, we introduce $\sigma$-modification to design the update law $\dot{\hat{\Psi}}_{i}$ :

$$
\dot{\hat{\Psi}}_{i}=\Gamma_{\Psi}\left(Z_{i, 1} x_{i, 2 c}^{T}(t) A_{i}^{T}(t)-\sigma_{\Psi} \widehat{\Psi}_{i}\right),
$$

where $\Gamma_{\Psi}$ is the gain of the adaptive law of $\widehat{\Psi}_{i}, \sigma_{\Psi}>0$.
3.2. Second-Order Disturbance Observer-Based Outer Loop Adaptive Antidisturbance Formation Control Law Design. In this section, to approximate the unknown uncertainties $\Delta f(\theta, v)_{i}$, we introduce a B-RBFNN $\widehat{\Theta}_{i}^{T} \Phi_{i}(x)$. Hence, it can be obtained that $\Delta f(\theta, v)_{i}=\Theta_{i}^{T} \Phi_{i}(x)+\varepsilon_{i}$, where $\varepsilon_{i}$ is the bounded estimation error. Let $\widehat{\Theta}_{i}$ be the estimations of $\Theta_{i}$.

In order to deal with the time-varying disturbances, the second-order disturbance observer for the control system (3) is designed as

$$
\begin{aligned}
\widehat{d}_{i} & =P_{i, 1}+l_{1} x_{i, 2}, \\
\dot{P}_{i, 1} & =-l_{1}\left(g\left(\alpha, \beta, \gamma_{s}\right)_{i} u_{i}(t)+f(\theta, v)_{i}+\widehat{\Theta}_{i}^{T} \Phi_{i}(x)+\widehat{d}_{i}(t)\right)+\hat{\dot{d}}_{i}, \\
\hat{\dot{d}}_{i} & =P_{i, 2}+l_{2} x_{i, 2}, \\
\dot{P}_{i, 2} & =-l_{2}\left(g\left(\alpha, \beta, \gamma_{s}\right)_{i} u_{i}(t)+f(\theta, v)_{i}+\widehat{\Theta}_{i}^{T} \Phi_{i}(x)+\widehat{d}_{i}(t)\right),
\end{aligned}
$$

where $\widehat{d}_{i}$ and $\hat{\dot{d}}_{i}$ represent the estimation of $d_{i}$ and $\dot{d}_{i}$, respectively; $P_{i, 1}$ and $P_{i, 2}$ are auxiliary variables; $l_{1}=\operatorname{diag}\left\{l_{11}, l_{12}, l_{13}\right\}$ and $l_{2}=\operatorname{diag}\left\{l_{21}, l_{22}, l_{23}\right\}$ are designed parameters.

From (2) and (7), we can obtain that

$$
\dot{Z}_{i, 2}=g\left(\alpha, \beta, \gamma_{s}\right)_{i} u_{i}(t)+f(\theta, v)_{i}+\Delta f(\theta, v)_{i}+d_{i}(t)-\dot{\beta}_{i, 1} .
$$

Therefore, considering the features of (15), the outer loop adaptive antidisturbance formation control law is designed as

$$
u_{i}(t)=g^{-1}\left(\alpha, \beta, \gamma_{s}\right)_{i}\left[-K_{2} Z_{i, 2}+\dot{\beta}_{i, 1}-\widehat{d}_{i}(t)-f(\theta, v)_{i}-\widehat{\Theta}_{i}^{T} \Phi_{i}(x)\right],
$$

where $K_{2} \in \mathbb{R}^{3 \times 3}$ represents the control gain of the outer loop.

Moreover, the update law of $\dot{\hat{\Theta}}$ on the basis of the $\sigma$-modification method is designed as

$$
\dot{\widehat{\Theta}}_{i}=\Gamma_{\Theta}\left(\Phi_{i}(x) Z_{i, 2}^{T}-\sigma_{\Theta} \widehat{\Theta}_{i}\right),
$$

where $\Gamma_{\Theta}$ is the gain of the adaptive law of $\widehat{\Theta}_{i}, \sigma_{\Theta}>0$.

The convergence property of the intelligent adaptive antidisturbance formation control laws can be analyzed by the following theorem.

Theorem 1. Consider the closed-loop formation control system consisting of the CAUS system (2), the antidisturbance control law (10), (11), and (16), the second order disturbance observer (14), and the adaptive update laws (13) and (17). Suppose Assumptions $1 \sim 2$ are satisfied. It can be guaranteed that all the signals are bounded and the positions of the followers can track the leader coordinately and accurately in spite of the multiple uncertainties.

Proof. Define the estimation error as 


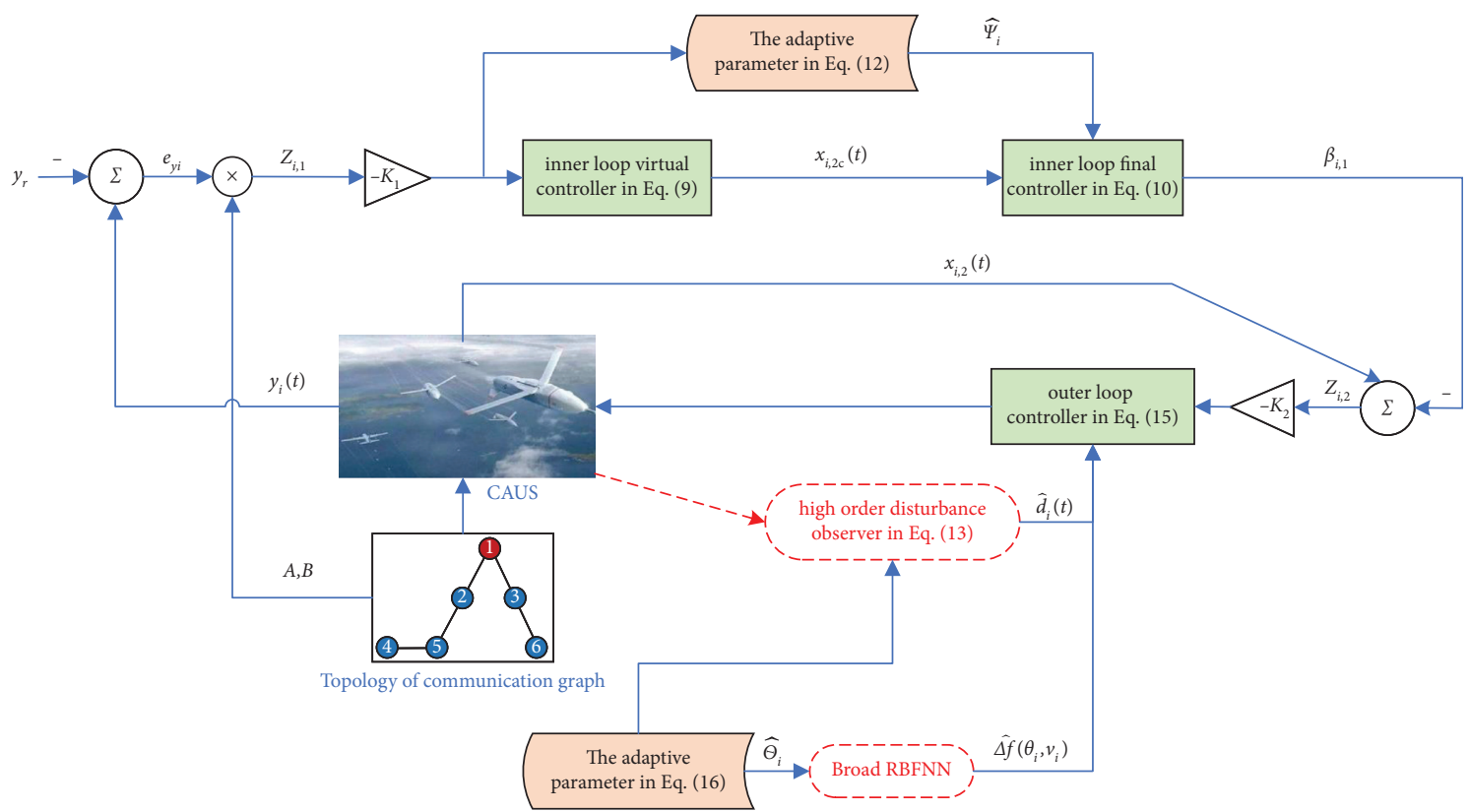

FIGURE 1: B-RBFNN-based adaptive formation control scheme structure for CAUS.

$$
\begin{aligned}
\widetilde{\zeta}_{d, i} & =\left[\widetilde{d}_{i}, \widetilde{\dot{d}}_{i}\right]^{T}, \\
\widetilde{d}_{i} & =d_{i}-\widehat{d}_{i}, \\
\widetilde{\dot{d}}_{i} & =\dot{d}_{i}-\widehat{\dot{d}}_{i},
\end{aligned}
$$

where $\widetilde{d}_{i}$ is the disturbance estimation error and $\widetilde{\dot{d}}_{i}$ represent the disturbance differential estimation error. Combining (2), (14), and $\widetilde{d}_{i}=d_{i}-\widehat{d}_{i}$, we can obtain that the differential of $\widetilde{d}_{i}$ as

$$
\begin{aligned}
\dot{\vec{d}}_{i} & =\dot{d}_{i}-\left(l_{1} \tilde{d}_{i}-l_{1} \widetilde{\Theta}_{i}^{T} \Phi_{i}(x)+l_{1} \varepsilon_{i}+\widehat{\dot{d}}_{i}\right) \\
& =-l_{1} \widetilde{d}_{i}+\tilde{\dot{d}}_{i}+l_{1} \widetilde{\Theta}_{i}^{T} \Phi_{i}(x)-l_{1} \varepsilon_{i} .
\end{aligned}
$$

In the similar way, according to $\widetilde{\dot{d}}_{i}=\dot{d}_{i}-\widehat{\dot{d}}_{i}$, we can get the differential of $\dot{d}_{i}$ that

$$
\dot{\dot{d}}_{i}=-l_{2} \tilde{d}_{i}+\ddot{d}_{i}+l_{2} \widetilde{\Theta}_{i}^{T} \Phi_{i}(x)-l_{2} \varepsilon_{i},
$$

where $\widetilde{\Theta}_{i}=\widehat{\Theta}_{i}-\Theta_{i}$. Substitute (20) into the differential of (19), we can easily know that

$$
\begin{aligned}
\ddot{\widetilde{d}}_{i} & =-l_{1} \dot{\vec{d}}_{i}+\dot{\vec{d}}_{i}+l_{1} \dot{\widehat{\Theta}}_{i}^{T} \Phi_{i}(x)-l_{1} \dot{\varepsilon}_{i} \\
& =-l_{1} \dot{\vec{d}}_{i}-l_{2} \tilde{d}_{i}+\ddot{d}_{i}+l_{1} \dot{\widehat{\Theta}}_{i}^{T} \Phi_{i}(x)+l_{2} \widetilde{\Theta}_{i}^{T} \Phi_{i}(x)-l_{1} \dot{\varepsilon}_{i}-l_{2} \varepsilon_{i} .
\end{aligned}
$$

According to (18)-(20), we can get that

$$
\dot{\bar{\zeta}}_{d, i}=A_{d, i} \widetilde{\zeta}_{d, i}+F_{d, i} \ddot{d}_{i}+L_{d, i}\left(\widetilde{\Theta}_{i}^{T} \Phi_{i}(x)-\varepsilon_{i}\right) \text {, }
$$

where

$$
\begin{aligned}
A_{d, i} & =\left[\begin{array}{cc}
-l_{1} & I^{3} \\
-l_{2} & 0
\end{array}\right], \\
F_{d, i} & =\left[\begin{array}{c}
0 \\
I^{3}
\end{array}\right], \\
L_{d, i} & =\left[\begin{array}{l}
l_{1} \\
l_{2}
\end{array}\right] .
\end{aligned}
$$

Clearly, we can always find the disturbance observer gains $l_{1}$ and $l_{2}$ such that there exists a symmetric positive definite matrix $Q_{d, i}$, whose minimum feature value is $\gamma_{m, i}$, and a symmetric positive definite matrix $P_{i}$ satisfies

$$
A_{d, i}^{T} P_{i}+P_{i} A_{d, i}=-Q_{d, i}
$$

Substitute (10)-(12) into (9), we can get that

$$
\dot{e}_{y i}=-K_{1} Z_{i, 1}-A_{i}(t) \Omega_{i} \widetilde{\Psi}_{i} x_{i, 2 c}(t)+A_{i}(t) \Omega_{i} Z_{i, 2},
$$

where $\widetilde{\Psi}_{i}=\widehat{\Psi}_{i}-\Psi_{i}$. Substitute (16) into (15) yields

$$
\dot{Z}_{i, 2}=-K_{2} Z_{i, 2}+\tilde{d}_{i}(t)-\widetilde{\Theta}_{i}^{T} \Phi_{i}(x)+\varepsilon_{i},
$$

where $\widetilde{\Theta}_{i}=\widehat{\Theta}_{i}-\Theta_{i}$. Thus, in order to prove the stability of this control system, we can take the Lyapunov candidate function as

$$
\begin{aligned}
V= & \sum_{i=1}^{n}\left(\widetilde{\zeta}_{d, i}^{T} P_{i} \widetilde{\zeta}_{d, i}+\frac{1}{2} e_{y i}^{T}(\mathscr{L}+\mathscr{B}) e_{y i}+\frac{1}{2 \Gamma_{\Psi}} \operatorname{tr}\left(\widetilde{\Psi}_{i}^{T} \Omega_{i} \widetilde{\Psi}_{i}\right)\right) \\
& +\sum_{i=1}^{n}\left(\frac{1}{2} Z_{i, 2}^{T} Z_{i, 2}+\frac{1}{2} \widetilde{d}_{i}^{T} \widetilde{d}_{i}+\frac{1}{2 \Gamma_{\Theta}} \operatorname{tr}\left(\widetilde{\Theta}_{i}^{T} \widetilde{\Theta}_{i}\right)\right)
\end{aligned}
$$


where $\operatorname{tr}(\cdot)$ represents the trace of matrices, $\mathscr{L}$ and $\mathscr{B}$ are the Laplacian matrix and the information connection matrix of the algebraic graph theory, respectively, which satisfy that $z_{i, 1}=(\mathscr{L}+\mathscr{B}) e_{y, i}$. Then, we can get the differential of (27) that

$$
\dot{V}=\sum_{i=1}^{n}\left(2 \widetilde{\zeta}_{d, i}^{T} P_{i} \dot{\tilde{\zeta}}_{d, i}+Z_{i, 1}^{T} \dot{e}_{y i}+\frac{1}{\Gamma_{\Psi}} \operatorname{tr}\left(\widetilde{\Psi}_{i}^{T} \Omega_{i} \dot{\hat{\Psi}}_{i}\right)\right)+\sum_{i=1}^{n}\left(Z_{i, 2}^{T} \dot{Z}_{i, 2}+\widetilde{d}_{i}^{T} \dot{\vec{d}}_{i}+\frac{1}{\Gamma_{\Theta}} \operatorname{tr}\left(\widetilde{\Theta}_{i}^{T} \dot{\hat{\Theta}}_{i}\right)\right)
$$

Substitute (13), (17), (22), (25), and (26) into (28), we know that

$$
\begin{aligned}
\dot{V}= & \sum_{i=1}^{n} 2 \widetilde{\zeta}_{d, i}^{T} P_{i}\left[A_{d, i} \widetilde{\zeta}_{d, i}+F_{d, i} \ddot{d}_{i}+L_{d, i}\left(\widetilde{\Theta}_{i}^{T} \Phi_{i}(x)-\varepsilon_{i}\right)\right] \\
& +\sum_{i=1}^{n} Z_{i, 1}^{T}\left(-K_{1} Z_{i, 1}-A_{i}(t) \Omega_{i} \widetilde{\Psi}_{i} x_{i, 2 c}(t)+A_{i}(t) \Omega_{i} Z_{i, 2}\right)+\frac{1}{\Gamma_{\Psi}} \operatorname{tr}\left(\widetilde{\Psi}_{i}^{T} \Omega_{i} \dot{\hat{\Psi}}_{i}\right) \\
& +\sum_{i=1}^{n} Z_{i, 2}^{T}\left(-K_{2} Z_{i, 2}+\widetilde{d}_{i}(t)-\widetilde{\Theta}_{i}^{T} \Phi_{i}(x)+\varepsilon_{i}\right)+\widetilde{d}_{i}^{T}\left(-l_{1} \tilde{d}_{i}+\tilde{\dot{d}}_{i}+l_{1} \widetilde{\Theta}_{i}^{T} \Phi_{i}(x)-l_{1} \varepsilon_{i}\right)+\frac{1}{\Gamma_{\Theta}} \operatorname{tr}\left(\widetilde{\Theta}_{i}^{T} \dot{\Theta}_{i}\right) .
\end{aligned}
$$

Since for any constant vector $a, b, \operatorname{tr}\left(a^{T} b\right)=b a^{T}$ holds, we can get the following inequality based on Assumption 1 , (22), and (24):

$$
\begin{aligned}
& 2 \widetilde{\zeta}_{d, i}^{T} P_{i}\left(A_{d, i} \widetilde{\zeta}_{d, i}+F_{d, i}+L_{d, i}\left(\widetilde{\Theta}_{i}^{T} \Phi_{i}(x)-\varepsilon_{i}\right)\right)=\widetilde{\zeta}_{d, i}^{T}\left(A_{d, i}^{T} P_{i}+P_{i} A_{d, i}\right) \widetilde{\zeta}_{d, i}+2 \widetilde{\zeta}_{d, i}^{T} P_{i} F_{d, i}+2 \widetilde{\zeta}_{d, i}^{T} P_{i} L_{d, i}\left(\widetilde{\Theta}_{i}^{T} \Phi_{i}(x)-\varepsilon_{i}\right) \\
& -\widetilde{\zeta}_{d, i}^{T} Q_{d, i} \widetilde{\zeta}_{d, i}+2\left\|\widetilde{\zeta}_{d, i}\right\|\left\|P_{i} F_{d, i}\right\| \mu_{i}+2\left\|\widetilde{\zeta}_{d, i}\right\|\left\|P_{i} L_{d, i}\right\| \chi_{m, i} \\
& \leq-\gamma_{m, i}\left\|\widetilde{\zeta}_{d, i}\right\|^{2}+\frac{2}{c_{i}}\left\|\widetilde{\zeta}_{d, i}\right\|^{2}+c_{i} \mu_{i}^{2}\left\|P_{i} F_{d, i}\right\|^{2}+c_{i} \chi_{m, i}^{2}\left\|P_{i} L_{d, i}\right\|^{2} \\
& \leq-\left(\gamma_{m, i}-\frac{2}{c_{i}}\right)\left\|\widetilde{\zeta}_{d, i}\right\|^{2}+c_{i}\left(\mu_{i}^{2}\left\|P_{i} F_{d, i}\right\|^{2}+\chi_{m, i}^{2}\left\|P_{i} L_{d, i}\right\|^{2}\right),
\end{aligned}
$$

where $c_{i}$ is a positive constant and $\chi_{m, i}$ represents the boundness of estimation error, that is, $\left\|\Delta \widehat{f}_{i}-\Delta f_{i}\right\|=\widetilde{\Theta}_{i}^{T} \Phi_{i}(x)-\varepsilon_{i} \leq \chi_{m, i}$.

$$
\begin{aligned}
\dot{V} \leq & \sum_{i=1}^{n}\left(-\left(\gamma_{m, i}-\frac{2}{c_{i}}\right)\left\|\widetilde{\zeta}_{d, i}\right\|^{2}+c_{i}\left(\mu_{i}^{2}\left\|P_{i} F_{d, i}\right\|^{2}+\chi_{m, i}^{2}\left\|P_{i} L_{d, i}\right\|^{2}\right)\right) \\
& +\sum_{i=1}^{n}\left(-Z_{i, 1}^{T} K_{1} Z_{i, 1}-Z_{i, 2}^{T} K_{2} Z_{i, 2}\right)+\sum_{i=1}^{n}\left(Z_{i, 1}^{T} A_{i}(t) \Omega_{i} Z_{i, 2}\right) \\
& +\sum_{i=1}^{n}\left(Z_{i, 2}^{T} \widetilde{d}_{i}(t)\right)+\sum_{i=1}^{n}\left(-\widetilde{d}_{i}^{T} l_{1} \widetilde{d}_{i}+\widetilde{d}_{i}^{T} \widetilde{d}_{i}\right)+\sum_{i=1}^{n}\left(Z_{i, 2}^{T}-\widetilde{d}_{i}^{T} l_{1}\right) \varepsilon_{i}+\sum_{i=1}^{n}\left(\widetilde{d}_{i}^{T} l_{1} \widetilde{\Theta}_{i}^{T} \Phi_{i}(x)\right) \\
& +\sum_{i=1}^{n}\left(-\sigma_{\Psi} \operatorname{tr}\left(\widetilde{\Psi}_{i}^{T} \Omega_{i} \widehat{\Psi}_{i}\right)\right)+\sum_{i=1}^{n}\left(-\sigma_{\Theta} \operatorname{tr}\left(\widetilde{\Theta}_{i}^{T} \widehat{\Theta}_{i}\right)\right) .
\end{aligned}
$$


Obviously, apply Young inequality, we can finally get that

$$
\begin{aligned}
\dot{V} & \leq-\sum_{i=1}^{n}\left(\gamma_{m, i}-\frac{2}{c_{i}}\right) \widetilde{\zeta}_{d, i}{ }^{2}+\sum_{i=1}^{n}\left(-Z_{i, 1}^{T} K_{1} Z_{i, 1}-Z_{i, 2}^{T}\left(K_{2}-\frac{1}{2} H_{i}^{T} H_{i}-\frac{1}{2} I-\frac{1}{4 c} I\right) Z_{i, 2}\right) \\
& +\sum_{i=1}^{n}\left(-\widetilde{d}_{i}^{T}\left(l_{1}-\frac{1}{4} I\right) \widetilde{d}_{i}\right)+\sum_{i=1}^{n}\left(-\frac{1}{2} \sigma_{\Theta} \operatorname{tr}\left(\widetilde{\Theta}_{i}^{T} \widetilde{\Theta}_{i}\right)-\frac{1}{2} \sigma_{\Psi} t r\left(\widetilde{\Psi}_{i}^{T} \Omega_{i} \widetilde{\Psi}_{i}\right)\right)+\tau_{i},
\end{aligned}
$$

where $H_{i}=Z_{i, 1}^{T} A_{i}(t)$ and

$$
\begin{aligned}
\tau= & \sum_{i=1}^{n} c_{i}\left(\mu_{i}^{2}\left\|P_{i} F_{d, i}\right\|^{2}+\chi_{m, i}^{2}\left\|P_{i} L_{d, i}\right\|^{2}\right) \\
& +\sum_{i=1}^{n}\left(\frac{1}{2} \lambda_{\max ^{2}}\left(\Omega_{i}\right)+c_{i}\left\|\widetilde{\Theta}_{i}\right\|^{2} \bar{\phi}_{m}^{2}+\widetilde{\dot{d}}_{i}^{T} \widetilde{\dot{d}}_{i}+\frac{1}{2} \sigma_{\Theta} \operatorname{tr}\left(\Theta_{i}^{T} \Theta_{i}\right)+\frac{1}{2} \sigma_{\Psi} \operatorname{tr}\left(\Psi_{i}^{T} \Omega_{i} \Psi_{i}\right)\right) .
\end{aligned}
$$

Combining (27) and (32), it is obvious that

$$
\dot{V} \leq-\lambda V+\tau \text {, }
$$

$$
\lambda=\min \left\{\begin{array}{lll}
\sum_{i=1}^{n} \frac{\left(\gamma_{m, i}-2 / c_{i}\right)}{\lambda_{\max }\left(P_{i}\right)}, & \frac{\lambda_{\max }(\mathscr{L}+\mathscr{B})^{-1}}{\lambda_{\max }\left(K_{1}\right)}, & \lambda_{\min }\left(2 l_{1}-\frac{1}{2} I\right) \\
\sum_{i=1}^{n} \lambda_{\min }\left(2 K_{2}-H_{i}^{T} H_{i}-I-\frac{1}{2 c} I\right), & \Gamma_{\Theta} \sigma_{\Theta}, \quad \Gamma_{\Psi} \sigma_{\Psi}
\end{array}\right\}
$$

So, the solutions of the CAUS $\widetilde{\zeta}_{d, i}, Z_{i, 1}, \widetilde{\Psi}_{i}, Z_{i, 2}, \widetilde{d}_{i}, \widetilde{\Theta}_{i}$, $i=1,2, \ldots, n$ are all bounded, which completes the proof.

\section{Simulation}

In this part, in order to verify the achievement of the trajectory coordination of CAUS with the proposed intelligent adaptive antidisturbance formation control method, the simulation with the CAUS model described by (2) is carried out.

It is assumed that the first AUS is the virtual leader; meanwhile, we design the topological graph of AUS as Figure 2. The simulation time is $T=15 \mathrm{~s}$. The mass of single AUS is $m=45 \mathrm{~kg}$. The parameters of the disturbance observer can be chosen as $l_{1}=\operatorname{diag}\{10,10,10\}$ and $l_{2}=\operatorname{diag}\{20,20,20\}$, and the gain of the inner loop controller and the outer loop controller are $k_{1}=\operatorname{diag}\{4,2,4\}$ and $k_{2}=\operatorname{diag}\{8,4,8\}$. The initial states and the desired states of CAUS are shown in Table 1: and

The uncertainties can be identified as $\Delta A_{i}=\operatorname{diag}\{1,1,1\}$ where 


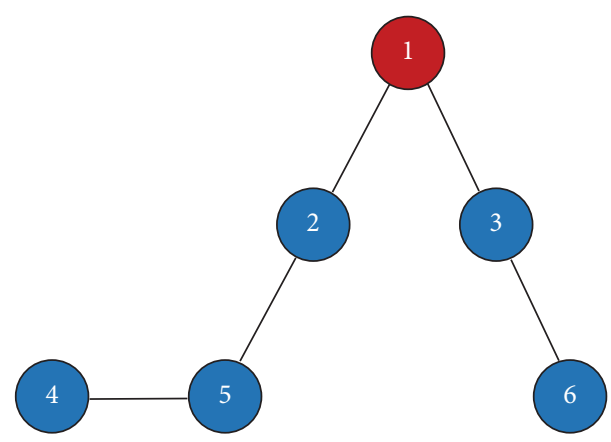

FIGURE 2: Topology of communication graph for CAUS.

TABle 1: The initial states and the desired states of CAUS.

\begin{tabular}{|c|c|c|c|c|c|c|}
\hline State/Number & 1 & 2 & 3 & 4 & 5 & 6 \\
\hline$x_{10}=\left[x_{i, 0}, y_{i, 0}, z_{i, 0}\right]^{T}$ & {$[10,20,10]^{T}$} & {$[10,20,10]^{T}$} & {$[10,20,10]^{T}$} & {$[10,20,10]^{T}$} & {$[10,20,10]^{T}$} & {$[10,20,10]^{T}$} \\
\hline$x_{20}=\left[v_{i, 0}, \theta_{i, 0}, \psi_{s, i 0}\right]^{T}$ & {$[1,0,1]^{T}$} & {$[1,1,0]^{T}$} & {$[0.5,1,1]^{T}$} & {$[1,1,1]^{T}$} & {$[1,0.8,1]^{T}$} & {$[1,1,0.4]^{T}$} \\
\hline$x_{1 d}=\left[x_{i} d, y_{i} d, z_{i} d\right]^{T}$ & {$[10,20,10]^{T}$} & {$[9,22,10]^{T}$} & {$[11,22,10]^{T}$} & {$[7,24,10]^{T}$} & {$[8,24,10]^{T}$} & {$[12,24,10]^{T}$} \\
\hline
\end{tabular}

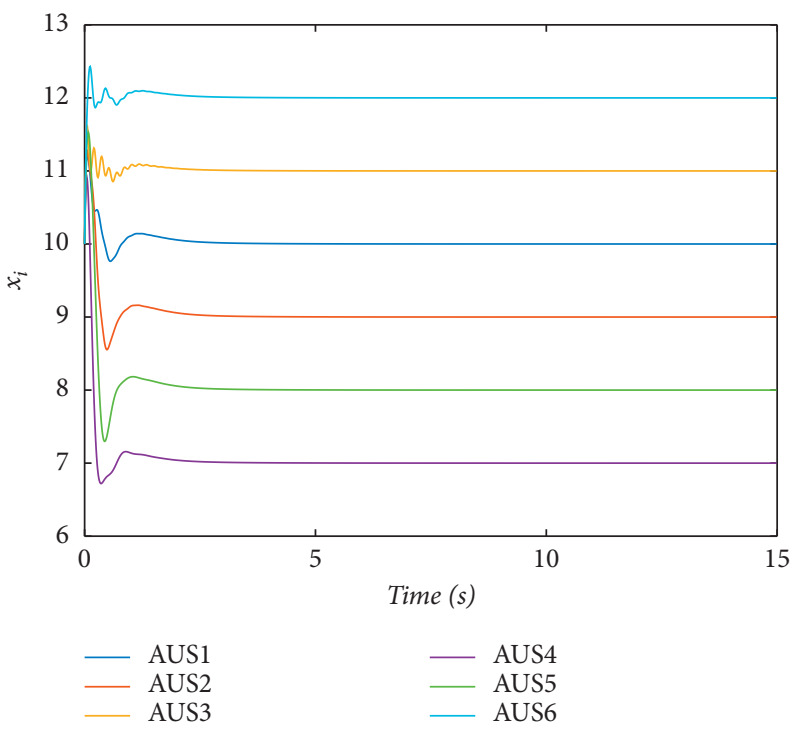

Figure 3: Trajectories of $x$ position of CAUS.

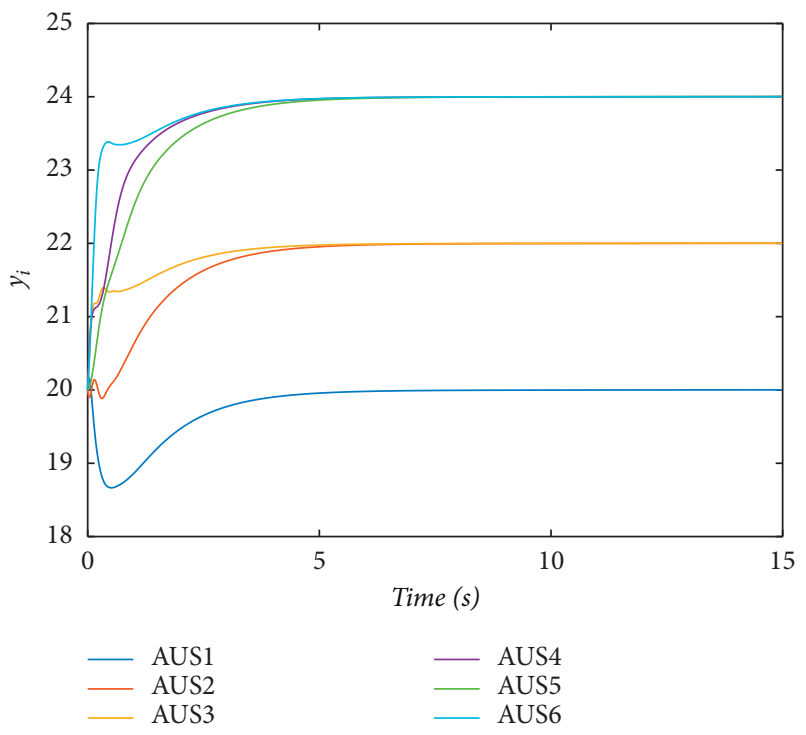

FIgURE 4: Trajectories of $y$ position of CAUS. 


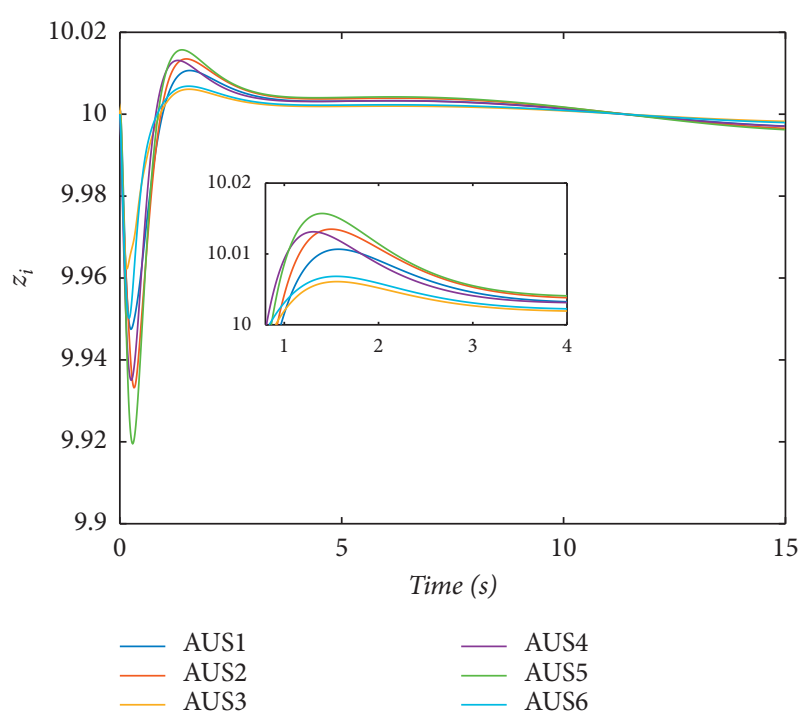

FIgURE 5: Trajectories of $z$ position of CAUS.
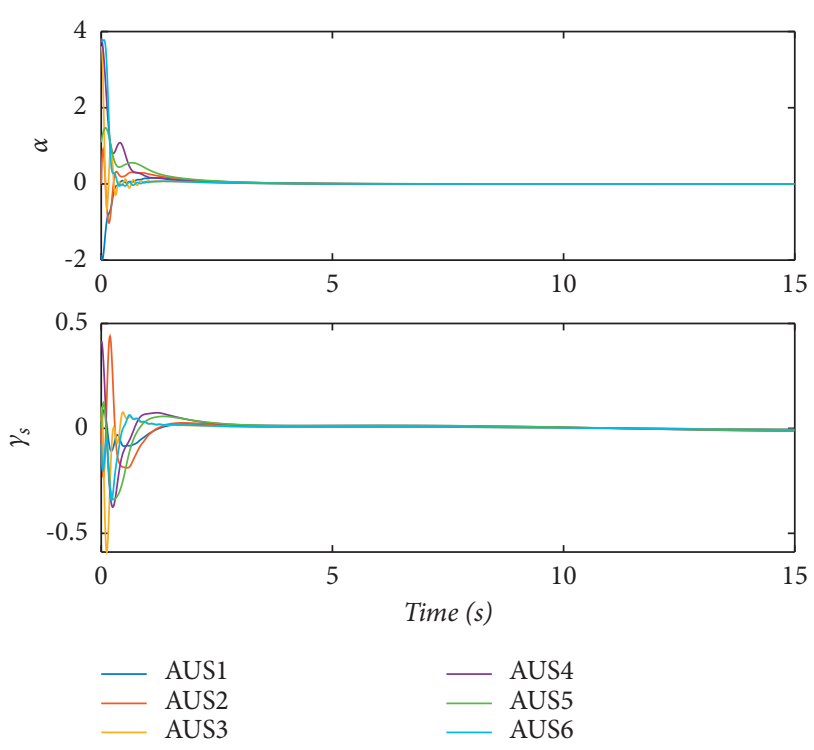

FIGURE 6: Trajectories of the angle of attack and the heeling angle of CAUS.

relative to the position of the leader according to the topological graph. Figure 6 shows the control attitude angles $\alpha$, $\gamma_{s}$ of every AUS can also be stable at a fixed value, respectively. From Figure 7, it is obvious that the boundedness of all the adaptive parameters can be guaranteed. The estimation of disturbances and the nonlinearities are shown in Figure 8. Figure 9 shows the characteristics of B-RBFNN, and the boundness of all the B-RBFNN nodes number can be verified. Figure 10 shows the trajectory of tracking errors of different control methods, which exhibits the advantage in control accuracy of the proposed method.

Remark 2. The selected results are obtained according to several rules, such as
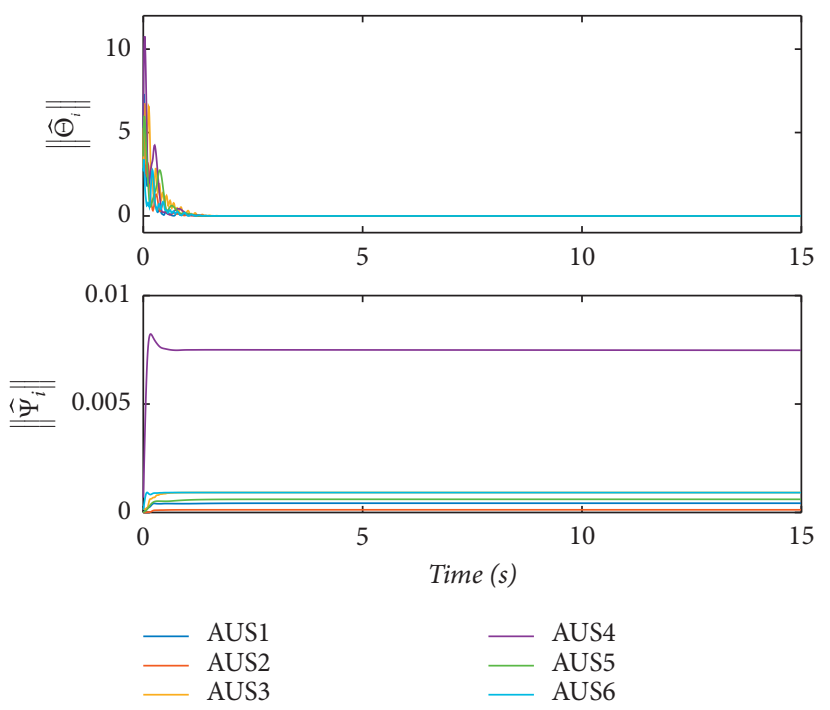

Figure 7: Trajectories of the adaptive parameters of CAUSs.
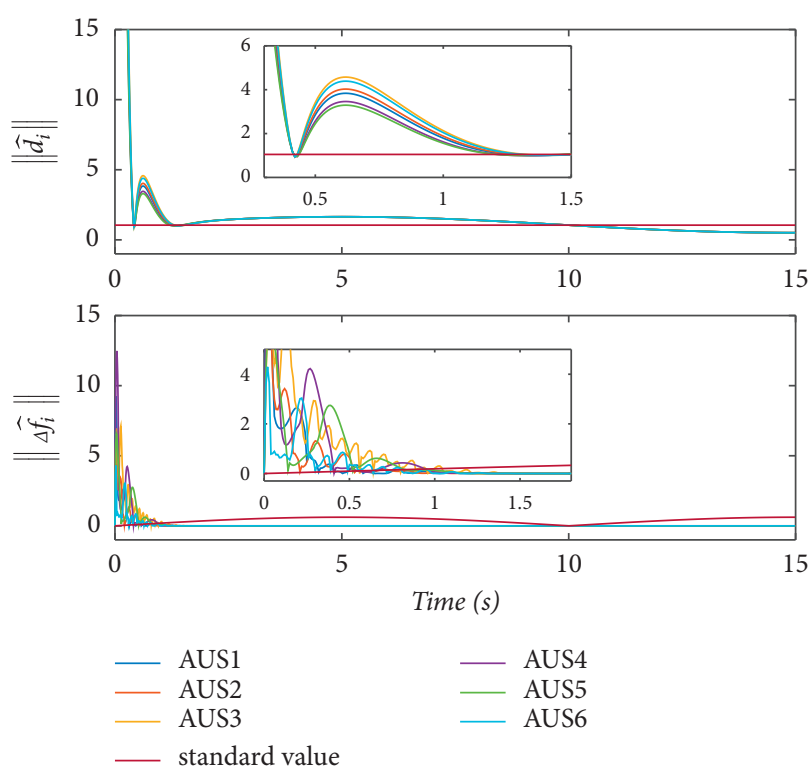

FIgURE 8: Trajectories of the estimation of $d_{i}$ and. $\Delta \hat{f}_{i}$.

(i) The control gains and the adaptive gains should be positive constants

(ii) Generally speaking, the control gains should satisfy that $k_{1} \leq 0.5 k_{2}, l_{1} \leq 0.5 l_{2}$

(iii) The adaptive damping parameters should satisfy that $\Gamma_{\Psi} \leq 0.001 \sigma_{\Psi}, \Gamma_{\Theta} \leq 30 \sigma_{\Theta}$

Based on the aforementioned rules, and through the adjustment and iteration on the basis of the simulation results, the design parameters can be finally selected.

\section{Conclusions}

In this paper, the B-RBFNN intelligence adaptive antidisturbance formation control method has been studied for 


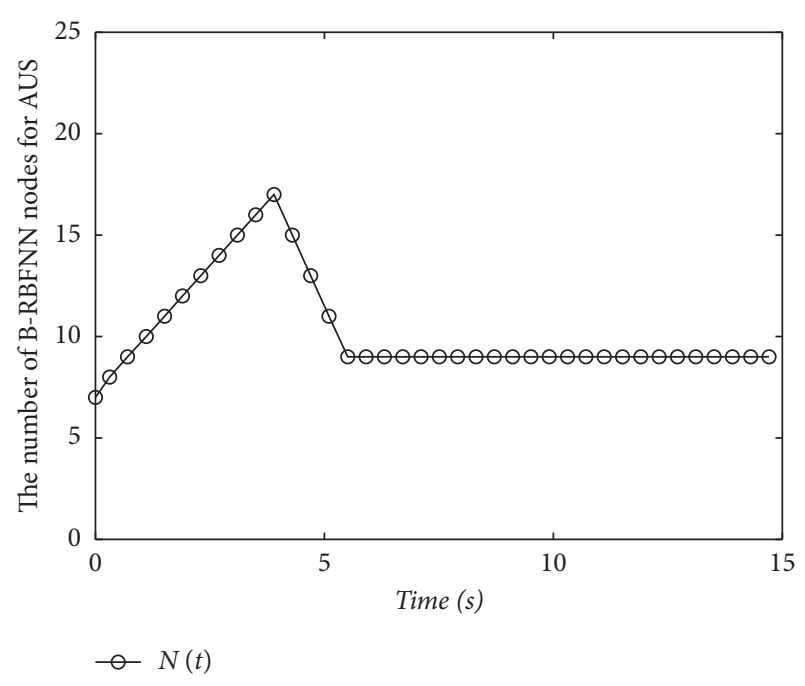

Figure 9: Trajectory of the B-RBFNN nodes number of CAUSs.

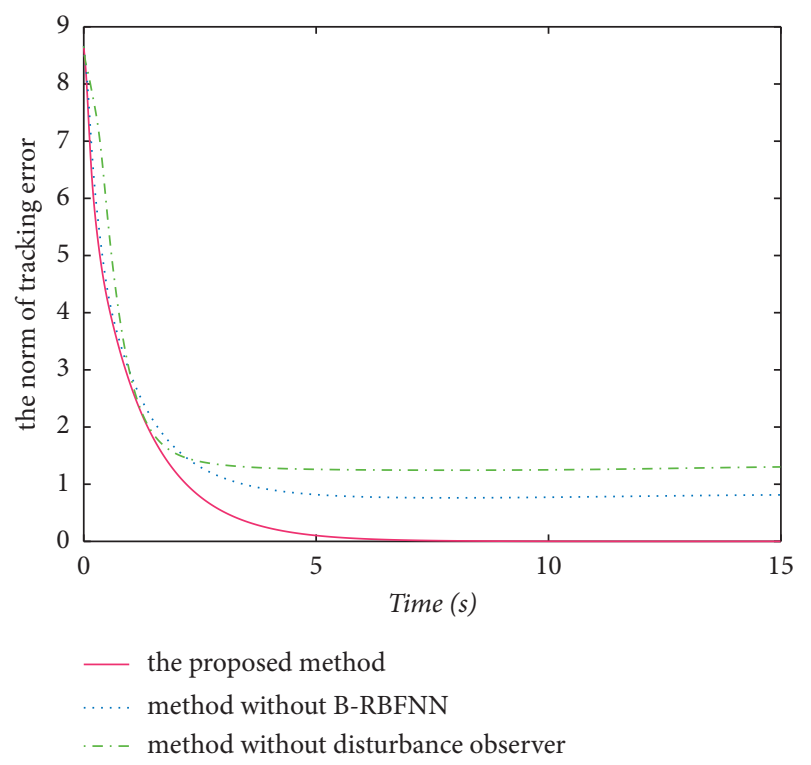

Figure 10: Trajectory of tracking errors of different control methods.

CAUSs in the presence of multi-source disturbances. According to the time-varying features of disturbances, a second-order disturbance observer which can both estimate the disturbance and its derivative, is designed. Meanwhile, the input-uncertainty-suppressed inner loop adaptive formation control law and the second order disturbance observer-based outer loop adaptive antidisturbance formation control law has been proposed. Furthermore, the introduction of B-RBFNN can maintain satisfactory approximation performance and achieve a rapid response rate for the formation control system of the CAUS. Meanwhile, the stability proof the closed-loop formation control system of the CAUS has been completed. A simulation example is given to verify the effectiveness of the proposed formation control scheme. In the future, we will consider the formation control issue of CAUS under the abruptly changing disturbances.

\section{Data Availability}

The data used to support the findings of this study are included within the article.

\section{Conflicts of Interest}

The authors declare that they have no conflicts of interest.

\section{Acknowledgments}

This work was supported in part by the National Natural Science Foundation of China under Grant no. 11772256 and sponsored by the Innovation Foundation for Doctor Dissertation of Northwestern Polytechnical University (G2017KY0412).

\section{References}

[1] C. Jiang, "Unmanned fighter aircraft on the future battlefield," Chinese Civil Air Defence, no. 5, pp. 5-7, 2003.

[2] C. Xia, L. Yongtai, Y. Liyuan, and Q. Lijie, "Cooperative task assignment and track planning for multi-uav attack mobile targets," Journal of Intelligent and Robotic Systems, vol. 300, no. 6.

[3] J.-f. Wang, G.-w. Jia, J.-c. Lin, and Z.-x. Hou, "Cooperative task allocation for heterogeneous multi-uav using multi-objective optimization algorithm," Journal of Central South University, vol. 27, no. 2, pp. 432-448, 2020.

[4] E. Wu, Y. Sun, J. Huang, C. Zhang, and Z. Li, "Multi uav cluster control method based on virtual core in improved artificial potential field," IEEE Access, vol. 8, no. 99, p. 1, 2020.

[5] J. Chen, Multi-robot system formation and experimental research, Ph.D. thesis, Beijing Institute of Technology, Beijing, China, 2015.

[6] D. B. Shen, Multi-robot formation control under limited leader speed information, Ph.D. thesis, South China University of Technology, Guangzhou, China, 2013.

[7] H. X. Qiu, H. B. Duan, and Y. M. Fan, "Multiple unmanned aerial vehicle autonomous formation based on the behavior mechanism in pigeon flocks," Control Theory \& Applications, no. 10, pp. 1298-1304, 2015.

[8] S. Wang, J. Zhang, and J. Zhang, "Artificial potential field algorithm for path control of unmanned ground vehicles formation in highway," Electronics Letters, vol. 54, no. 20, pp. 1166-1168, 2018.

[9] G. Lee and D. Chwa, "Decentralized behavior-based formation control of multiple robots considering obstacle avoidance," Intelligent Service Robotics, vol. 11, 2018.

[10] X. Cai and M. D. Queiroz, "Adaptive rigidity-based formation control for multirobotic vehicles with dynamics," IEEE Transactions on Control Systems Technology, vol. 23, no. 1, pp. 389-396, 2014.

[11] S. Jongho, K. Seungkeun, and S. Jinyoung, "Development of robust flocking control law for multiple uavs using behavioral decentralized method," Journal of The Korean Society for Aeronautical and Space Ences, vol. 43, no. 10, pp. 859-867, 2015. 
[12] A. Askari, M. Mortazavi, and H. A. Talebi, "Uav formation control via the virtual structure approach," Journal of Aerospace Engineering, vol. 28, no. 1, Article ID 04014047, 2015.

[13] M. Turpin, N. Michael, and V. Kumar, "Trajectory design and control for aggressive formation flight with quadrotors," Autonomous Robots, vol. 33, no. 1-2, pp. 143-156, 2012.

[14] M. Saska, T. Baca, J. Thomas et al., "System for deployment of groups of unmanned micro aerial vehicles in gps-denied environments using onboard visual relative localization," Autonomous Robots, vol. 41, no. 4, pp. 919-944, 2017.

[15] J. Shao, G. Xie, and L. Wang, "Leader-following formation control of multiple mobile vehicles," IET Control Theory \& Applications, vol. 1, no. 2, pp. 545-552, 2007.

[16] Y. Feng, X. Yu, and F. Han, "On nonsingular terminal slidingmode control of nonlinear systems," Automatica, vol. 49, no. 6, pp. 1715-1722, 2013.

[17] J. Song, Y. Niu, and Y. Zou, Finite-time Sliding Mode Control Synthesis under Explicit Output Constraint, Pergamon Press, Inc., Oxford, UK, 2016.

[18] G. Tao, "Multivariable adaptive control: a survey," Automatica, vol. 50, no. 11, pp. 2737-2764, 2014.

[19] T. Gibson, Z. Qu, A. Annaswamy, and E. Lavretsky, "Adaptive output feedback based on closed-loop reference models," IEEE Transactions on Automatic Control, vol. 60, no. 10, p. 1, 2014.

[20] E. Lavretsky and K. A. Wise, "Robust and adaptive control," Advanced Textbooks in Control \& Signal Processing, vol. 11, no. 96, pp. 367-372, 2012.

[21] W. Wei Kang, "Nonlinear H/sub $\infty /$ control and its application to rigid spacecraft," IEEE Transactions on Automatic Control, vol. 40, no. 7, pp. 1281-1285, 1995.

[22] S. Li, J. Yang, W. H. Chen, and X. Chen, Disturbance ObserverBased Control: Methods and Applications, CRC Press, Inc., Boca Raton, FL, USA, 2014.

[23] W. H. Chen, J. Yang, L. Guo, and S. Li, "Disturbance observerbased control and related methods: an overview," IEEE Transactions on Industrial Electronics, vol. 63, no. 2, p. 1, 2015.

[24] T. Jiang, C. Huang, and L. Guo, "Control of uncertain nonlinear systems based on observers and estimators," Automatica, vol. 59, pp. 35-47, 2015.

[25] B.-Z. Guo, "Active disturbance rejection control: from ODEs to PDEs ** this work was carried out with the support of the national natural science foundation of China and the national research foundation of South Africa," IFAC-PapersOnLine, vol. 49, no. 8, pp. 278-283, 2016.

[26] W.-H. Chen, "Disturbance observer based control for nonlinear systems," IEEE/ASME Transactions on Mechatronics, vol. 9, no. 4, pp. 706-710, 2004.

[27] X. S. Chen, J. Yang, S. H. Li, and Q. Li, "Disturbance observer based multi-variable control of ball mill grinding circuits," Journal of Process Control, vol. 19, no. 7, pp. 1205-1213, 2009.

[28] Q. C. Zhong, A. Kuperman, and R. K. Stobart, "Design of udebased controllers from their two-degree-of-freedom nature," International Journal of Robust \& Nonlinear Control, vol. 21, no. 17, pp. 1994-2008, 2011.

[29] H. Liu, L. Guo, and Y. Zhang, "An anti-disturbance pd control scheme for attitude control and stabilization of flexible spacecrafts," Nonlinear Dynamics, vol. 67, no. 3, pp. 2081-2088, 2012.

[30] L. Guo and W. Chen, "Disturbance attenuation and rejection for systems with nonlinearity via dobc approach," International Journal of Robust \& Nonlinear Control, vol. 15, no. 3, pp. 109-125, 2010.
[31] X. Yao and L. Guo, "Composite anti-disturbance control for Markovian jump nonlinear systems via disturbance observer," Automatica, vol. 49, no. 8, pp. 2538-2545, 2013.

[32] H. Sun and L. Guo, "Composite adaptive disturbance observer based control and back-stepping method for nonlinear system with multiple mismatched disturbances," Journal of the Franklin Institute, vol. 351, no. 2, pp. 1027-1041, 2014.

[33] X. Wei and N. Chen, "Composite hierarchical anti-disturbance control for nonlinear systems with dobc and fuzzy control," International Journal of Robust \& Nonlinear Control, vol. 24 , no. 2 .

[34] Z. Wang and J. Yuan, "Non-linear disturbance observer-based adaptive composite anti-disturbance control for non-linear systems with dynamic non-harmonic multisource disturbances," Transactions of the Institute of Measurement and Control, vol. 40, no. 12, pp. 3458-3465, 2017.

[35] Z. Wang and J. Yuan, "Dissipativity-based disturbance attenuation control for T-S fuzzy Markov jumping systems with nonlinear multisource uncertainties and partly unknown transition probabilities," IEEE Transactions on Cybernetics, no. 99, pp. 1-12, 2020.

[36] G. Zhu, J. Qi, and C. Wu, "Landing control of fixed-wing uav based on adrc," in Proceedings of the Chinese Control Conference, pp. 8020-8025, Guangzhou, China, July 2019.

[37] C. Yu, J. Lei, Li Tao, and G. Lei, "Robust tracking control for a quadrotor uav via dobc approach," in Proceedings of the 30th Chinese Control Decision, pp. 559-563, Shenyang, China, June 2018.

[38] B. Yu, S. Kim, and J. Suk, "Robust control based on adrc and dobc for small-scale helicopter," IFAC-PapersOnLine, vol. 52, no. 12, pp. 140-145, 2019.

[39] C. L. P. Chen and Z. Liu, "Broad learning system: an effective and efficient incremental learning system without the need for deep architecture," IEEE Transactions on Neural Networks and Learning Systems, vol. 29, no. 99, pp. 10-24, 2018.

[40] W. Hui, G. Cunfeng, W. Bolan, G. Longfei, and M. Weimin, "Algorithm and simulation of intelligent escape control for unmanned swarm," Journal of Command and Control, vol. 6, no. 2, pp. 83-88, 2020.

[41] Z. Wang, J. Yuan, Y. Pan, and J. Wei, "Neural network-based adaptive fault tolerant consensus control for a class of high order multiagent systems with input quantization and timevarying parameters," Neurocomputing, vol. 266, no. 29, pp. 315-324, 2017. 